\title{
Characterizing the Flow of Thickened Barium and Non-barium Liquid Recipes Using the IDDSI Flow Test
}

\author{
Carly E. A. Barbon ${ }^{1,2}$ (D) Catriona M. Steele ${ }^{1,2,3}$ \\ Received: 16 February 2018/ Accepted: 29 May 2018/ Published online: 11 June 2018 \\ (c) The Author(s) 2018
}

\begin{abstract}
The use of thickened liquids for dysphagia management has become wide-spread.Videofluoroscopy is commonly used to determine dysphagia severity and to evaluatethe effectiveness of interventions, including texture modification, but this requires theuse of radio-opaque contrast media. In order for the results of a videofluoroscopy tohave validity with respect to confirming swallowing safety and efficiency on differentliquid consistencies, it is important to understand the flow characteristics of the contrastmedia used and how the flow of these stimuli compares to the flow of liquids that areprovided outside the assessment context. In this study, we explored the flowcharacteristics of $20 \% \mathrm{w} / \mathrm{v}$ barium and non-barium stimuli prepared using starch andgum thickeners to reach the slightly, mildly and moderately thick liquid categoriesdefined by the International Dysphagia Diet Standardisation Initiative (IDDSI). Our goalwas to identify recipes that would produce stimuli with stable flow properties over a $3 \mathrm{~h}$ time frame post mixing. Thickener concentration was titrated to achieve matchingflow (i.e., IDDSI Flow Test results within a $1 \mathrm{ml}$ range) across the four stimulus types(non-barium starch, nonbarium gum, barium starch, barium gum) within each IDDSIlevel. The combination of barium and thickeners resulted in further thickening,particularly with starch-based thickening agents. A probe of the influence ofrefrigeration showed no difference in flow measures between chilled and roomtemperature stimuli over a 3-h time frame. Overall, recipes with stable flow overthree hours were identified for all barium and non-barium liquids tested.

Keyword · Deglutition · Deglutition disorders · Dysphagia · Texture modification · Videofluoroscopy · Thickened liquids · Barium
\end{abstract}

\section{Introduction}

Diet texture modification is widely recommended to promote and maintain patient safety when managing dysphagia [1]. Thickened liquids are used as an intervention based on evidence that boluses with higher viscosity travel more slowly through the oropharynx, and are less likely to be

Carly E. A. Barbon

carly.barbon@uhn.ca

1 Swallowing Rehabilitation Research Laboratory, Toronto Rehabilitation Institute-University Health Network, 550 University Avenue, 12th floor, Toronto, ON M5G 2A2, Canada

2 Department of Speech-Language Pathology, Faculty of Medicine, Rehabilitation Sciences Institute, University of Toronto, Toronto, ON, Canada

3 Board of Directors, International Dysphagia Diet Standardisation Initiative, Brisbane, Australia aspirated [2]. Despite the widespread use of thickened liquids, little is known regarding the specific flow characteristics that are needed to achieve therapeutic benefit [2]. Recent studies have also shown a risk of greater postswallow residue with extremely thick liquids [2]. In light of this evidence of potential risk as well as benefit with the use of thickened liquids, the effectiveness and optimal consistency of thickened liquids should be evaluated on a case by case basis using instrumental swallowing examinations.

In order for a bolus to be visualized in videofluoroscopy, a radiographic contrast agent must be used. In North America, barium is the most common contrast agent used. In the United States, there is one line of available barium products that comes in an array of different consistencies, specifically intended for imaging of the oropharynx

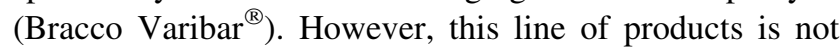
currently approved for clinical use outside the United States. Consequently, the standard of care in Canada and 
many other countries is for clinicians to prepare barium stimuli in different consistencies using off-label recipes. However, the addition of a thickening agent to a barium solution (or vice versa, the addition of barium powder to a pre-thickened liquid) may result in further thickening [3]. When this happens, the validity of the assessment stimulus for predicting swallowing function outside the context of the exam becomes a concern $[2,4,5]$.

In addition to the amount of thickener that is used in a recipe for a thickened liquid [6, 7], a number of other factors may influence the flow characteristics of the resulting stimulus. These factors have relevance for the preparation of thickened barium stimuli as well as nonbarium stimuli. Since the first descriptions of thickened liquids used for managing aspiration risk [8, 9], increasing varieties of thickeners and pre-thickened liquids have emerged on the market. Variations in liquid flow occur across different thickener types (e.g., modified corn starch versus xanthan gum) $[10,11]$. Other factors that may be relevant include thickening technique (e.g., hand-mixed versus machine-mixed) [12]; time post mixing [10, 11, 13]; temperature [13]; and characteristics of the liquid being thickened (e.g., pH, fat content, protein content, and volume of liquid in the recipe) [14, 15]. Non-linear concentration curves may be observed with some thickening agents [16].

Historically, guidelines regarding thickened liquids used in dysphagia management have defined categories of progressively thicker liquids in terms of viscosity [17, 18] (National Dysphagia Diet Task Force), most commonly reported at a shear rate of 50/s [17]. However, neither the equipment nor the expertise required to measure viscosity at controlled shear rates is accessible to clinicians. Recently, the International Dysphagia Diet Standardization Initiative (www.iddsi.org) has released new guidelines for classifying liquid thickness according to gravity flow through a syringe $[18,19]$. Three levels of thickened liquid (slightly thick, mildly thick, and moderately thick) are defined based on the height of the residual fluid column (in $\mathrm{ml}$ ) after $10 \mathrm{~s}$ of flow through a standard $10 \mathrm{ml}$ slip tip syringe (Becton-Dickinson manufacturer code 301604). A fourth level of extremely thick liquids shows no flow through the syringe; supplementary spoon tilt and fork drip tests are recommended to confirm the characteristics of liquids at this level.

In this article, we explore the flow characteristics of barium stimuli prepared according to recipes using two different commercial thickeners (modified corn starch and xanthan gum) in combination with water and barium sulfate powder, and compare the results of these tests to nonbarium stimuli prepared using water and the same thickening agents. The method of flow measurement selected for this study is the gravity-flow test recommended by the
International Dysphagia Diet Standardisation Initiative (IDDSI) [18, 19] The primary objective was to identify recipes for barium stimuli that would meet the IDDSI definitions of slightly, mildly, and moderately thick liquids [18] at $1-\mathrm{h}$ post mixing and remain stable within these defined flow ranges up to 3 -h post mixing. The process involved testing of different concentrations of thickener to determine the need to adjust the amount of thickener in a barium recipe compared to the amounts used when preparing non-barium stimuli. As an additional objective, we wanted to conduct a preliminary probe of the impact of temperature (i.e., room temperature vs. chilled) on the flow characteristics of thickened barium stimuli.

\section{Methods}

\section{Stimulus Mixing}

Thickened non-barium liquids were prepared using Nestlé ThickenUp $^{\circledR}$ (starch) and Nestlé ThickenUp ${ }^{\circledR}$ Clear $^{\circledR}$ (xanthan-gum) powders and a commercially available lemon-flavored water (Nestle ${ }^{\circledR}$ Lemon Splash). The nonbarium stimuli were developed for use in a related study of swallowing; lemon-flavored water was chosen to make these stimuli more palatable than thickened water. The taste of the unthickened Lemon Splash product was rated by a blinded taste panel, who judged the intensity of the sourness to be similar to a solution of $0.02 \%$ lemon juice and sweetness to $0.02 \%$ sucrose in water. This degree of sourness falls well below the levels reported to impact swallowing behaviors [20]. For the barium stimuli, the same thickening agents were mixed with a $20 \% \mathrm{w} / \mathrm{v}$ concentration barium suspension, comprising bottled water (Nestlé $^{\circledR}$ Pure Life) and Bracco E-Z-Paque ${ }^{\circledR} 96 \%$ w/w barium powder. All stimulus mixing was performed by a single research team member using a commercially available Bosch stand mixer (Model MUM4405UC, 4 speed, $400 \mathrm{~W}$ motor). Mixer speed was confirmed prior to the experiment by placing markers on the whisk, video recording the whisk in motion, and determining the number of rotations per second. The standard operating procedure for mixing was as follows:

(1) The desired amount of water was poured into the Bosch stand mixer mixing bowl, with the amount confirmed by weight on an OHAUS digital balance (model number PA1502 analytical scale: capacity: $1.5 \mathrm{~kg}$; readability $0.01 \mathrm{~g}$ ).

(2) The mixing bowl was removed, and a plastic nonstatic weigh boat ( $\mathrm{VWR}^{\circledR} 20.5 \mathrm{~cm}^{3}$ capacity) was placed on the balance. 
(3) The balance was tared to account for the weigh boat, and the thickener was added until the target weight was achieved.

(4) For the barium stimuli, the required amount of barium powder was similarly measured using a weigh boat.

(5) The water was set in motion using the Bosch stand mixer at a low spin speed of $60 \mathrm{rpm}$.

(6) The weighed barium powder was added to the water, while in motion.

(7) The weighed thickening powder was added, while the water was in motion. This process was completed slowly (over 10-20 s) in order to avoid clumping of the thickener.

(8) Once the thickener was added, the speed was increased by one level for $10 \mathrm{~s}$, and then lowered back to low speed.

(9) The liquid was then left to mix for a period of $1 \mathrm{~min}$ and $50 \mathrm{~s}$ (2-min mixing time overall) at a slow spin speed of $60 \mathrm{rpm}$.

(10) The liquid stimulus was portioned into separate, disposable cups with lids to allow for testing at subsequent timepoints.

Trials of various amounts of thickener were tested at the 1-h mark, beginning with the manufacturer instructed amount. If the liquid flow fell outside the targeted IDDSI range, iterative testing of small differences in thickener concentration was explored, as follows:

(1) Non-barium thickened with starch in concentrations of $4.1,4.15,4.2,4.75,4.77,4.8,5.0,5.5,5.8,6.0$, and $7.8 \mathrm{~g} / 100 \mathrm{ml}$.

(2) Non-barium thickened with xanthan gum in concentrations of $0.65,1.0,1.1,1.25,1.3,1.4$, and $2.1 \mathrm{~g} /$ $100 \mathrm{ml}$.

(3) Barium thickened with starch in concentrations of 2.64, 2.85, 2.87, 3.0, 3.3, 3.75, 3.8, 3.9, 5.1, 6.7, 7.4, and $7.6 \mathrm{~g} / 100 \mathrm{ml}$.

(4) Barium thickened with xanthan gum in concentrations of $0.35,0.5,0.9,1.0,1.02,1.05,2.0,2.2,4.2$, and $4.5 \mathrm{~g} / 100 \mathrm{ml}$.

\section{Flow Testing}

The IDDSI Flow Test was used to measure the flow properties of all liquid stimuli created for the study. Detailed instructions for this test can be found at: http:// iddsi.org/framework/drink-testing-methods/. All tests were conducted by a single research team member. Each stimulus was tested in triplicate at each measurement timepoint. Clean, fresh syringes were used for each sample. Stability in flow was considered to have been achieved when results across successive timepoints remained within a $1 \mathrm{ml}$ flow test result range. In the event that the observed range in flow test results across three repeated samples spanned more than $1 \mathrm{ml}$, a fourth sample was tested to correct for possible outlier results attributable to the possibility that small lumps or bubbles might have blocked the syringe nozzle. In these cases, the outlier result was discarded, and the three closest test results were retained for analysis. Similarity in flow across the different stimuli (barium + thickener combinations) was considered to have been achieved when results at the same timepoint fell within a flow test result range of $1 \mathrm{ml}$ across stimuli.

\section{Stability Testing}

To assess stability of the flow characteristics of the stimuli, we performed repeated testing over different timepoints (1-, 2-, and 3-h post mixing), with a goal to identify recipes that produced liquids with IDDSI Flow Test results that remained stable within IDDSI level over a timeframe of $3 \mathrm{~h}$.

\section{Temperature Testing}

Exploration of the impact of temperature was conducted using barium thickened with $0.35,0.5,1.0,2.0$, and $4.2 \mathrm{~g} /$ $100 \mathrm{ml}$ of xanthan gum and with 3, 3.3, 3.9, 5.1, and $6.7 \mathrm{~g} /$ $100 \mathrm{ml}$ of starch. The initial batch was divided into portions that were maintained either at room temperature or stored in a refrigerator (at approximately 4 degrees Celsius). Repeated flow tests were performed on both roomtemperature and chilled samples at 1-, 2-, and 3-h post mixing.

\section{Analyses}

Means and ranges for flow test results were calculated by thickener concentration $(\mathrm{g} / 100 \mathrm{ml})$ for each timepoint of measurement. Descriptive statistics and graphs will be used to illustrate the results of the flow testing.

For the demonstration of recipe stability, we conducted repeated measures ANOVAs of IDDSI Flow Test result within liquid type (barium/non-barium plus starch or gum) across time with a covariate of concentration. Similarly, for the exploration of temperature-based variations, we conducted a repeated measures ANOVA of IDDSI Flow Test result within liquid type (starch; gum) for matched chilled versus room-temperature barium recipes across timepoints with a covariate of concentration. 


\section{Results}

Trends in the flow test results across thickener concentrations at the 1-h timepoint post mixing can be seen in Fig. 1a-d. A clear pattern of higher flow test residual volumes (i.e., thicker liquids) is seen with higher concentrations of thickener within each liquid type. The stability analysis showed this result to be statistically significant for all four liquids, with $p$-values ranging from $<0.001$ to 0.002 .

Across time, none of the recipes showed a shift across IDDSI level boundaries. However, statistically significant increases in thickness within IDDSI level were found in pairwise comparisons between the 1 - and 2-h $(p=0.009)$ and the 2- and 3-h timepoints $(p=0.004)$ for the nonbarium starch-thickened stimuli. This effect was not found for the other liquids.

Figures 2 (starch) and 3 (gum) illustrate differences in flow of thickened barium between room-temperature and refrigerated samples across time. Although the refrigerated barium stimuli were thicker than the room-temperature stimuli, this difference was not statistically significant.
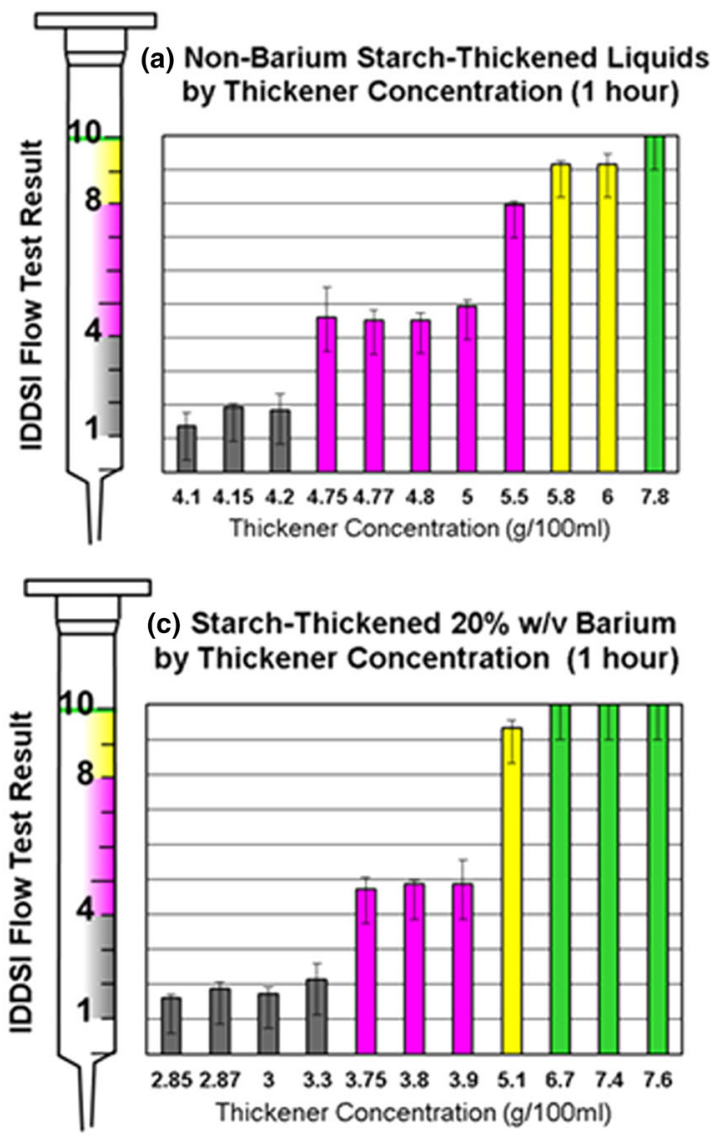

\section{Discussion}

The creation of assessment stimuli that have similar flow properties to non-barium liquids that are available for dysphagia management is important for patient care and diagnosis. The IDDSI Flow Test provides clinicians with a means of confirming similarity in flow across liquids. This study was designed to identify recipes for slightly, mildly, and moderately thick liquid barium stimuli that would remain stable at $1 \mathrm{~h}$ after mixing and up to $3 \mathrm{~h}$ afterward. Final recipes of the barium and non-barium stimuli can be found in Table 1. The results underscore the importance of clinicians developing awareness of factors that can contribute to variations in the flow of liquids and of methods for measuring flow. This is of particular relevance when preparing barium stimuli for use in videofluoroscopy.

The study highlights the flow of four different types of liquid, depending on thickener, and whether or not barium has been added. Figure 1a-d shows the decrease in flow that was seen as the amount of thickener was increased. It is important to highlight that the amount of thickener needed to reach any given level of thickness on the IDDSI

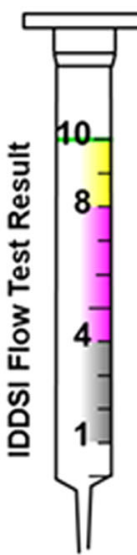

(b) Non-Barium Gum-Thickened Liquid by Thickener Concentration (1 hour)

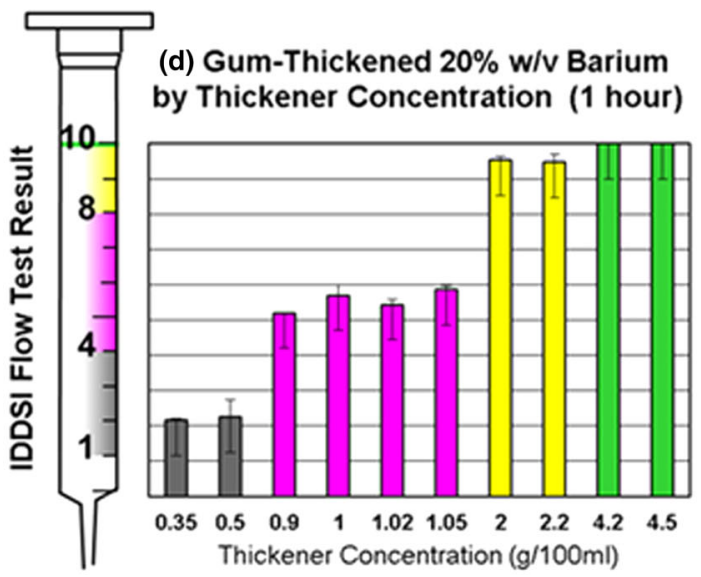

Fig. 1 IDDSI Flow Test results at $1 \mathrm{~h}$ for a non-barium starch, b non-barium xanthan gum, $\mathbf{c}$ starch-thickened barium, and $\mathbf{d}$ xanthan-gumthickened barium at $1-\mathrm{h}$ post mixing 


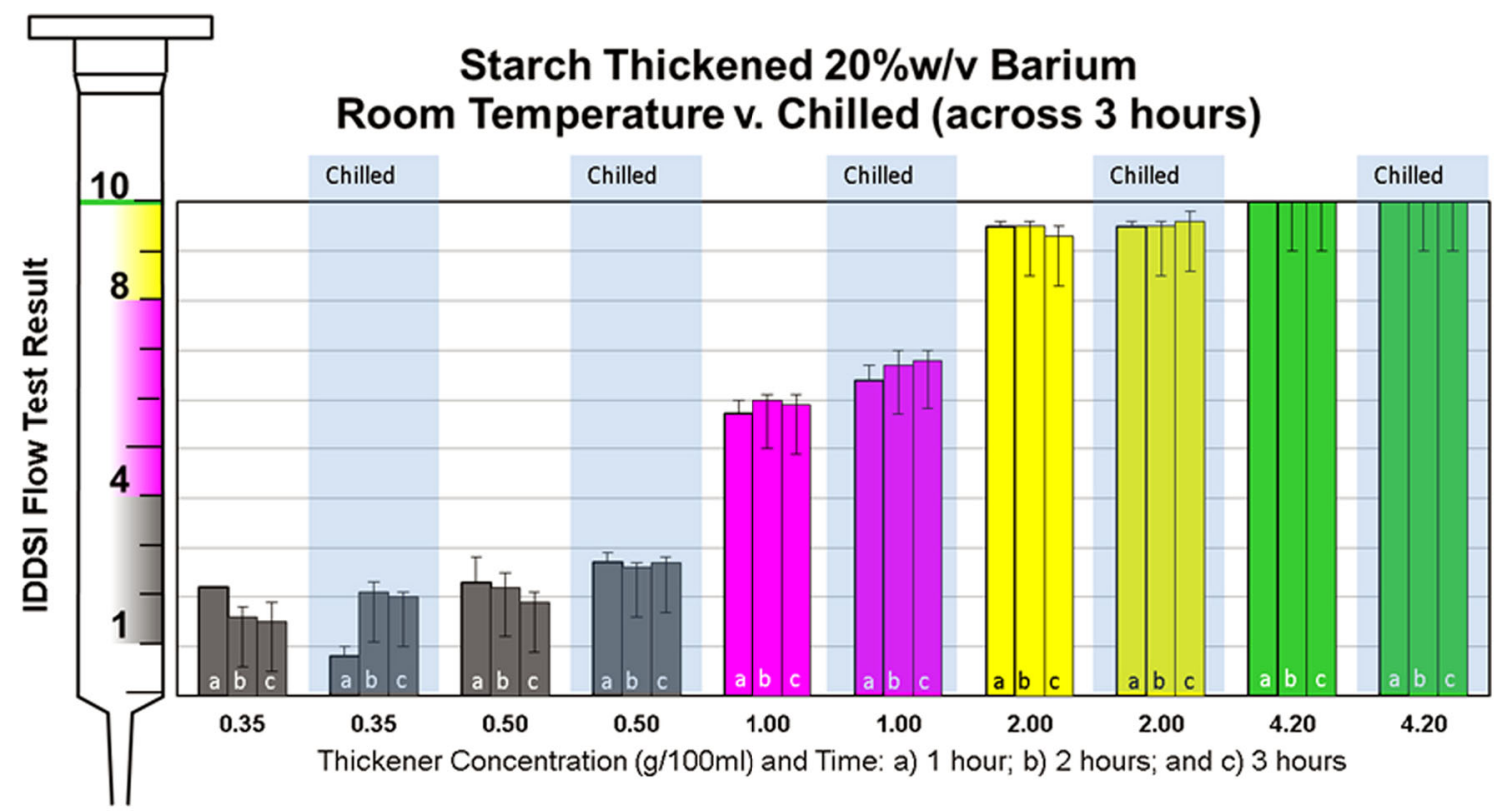

Fig. 2 IDDSI Flow Test results for room-temperature and chilled starch-thickened barium over $3 \mathrm{~h}$

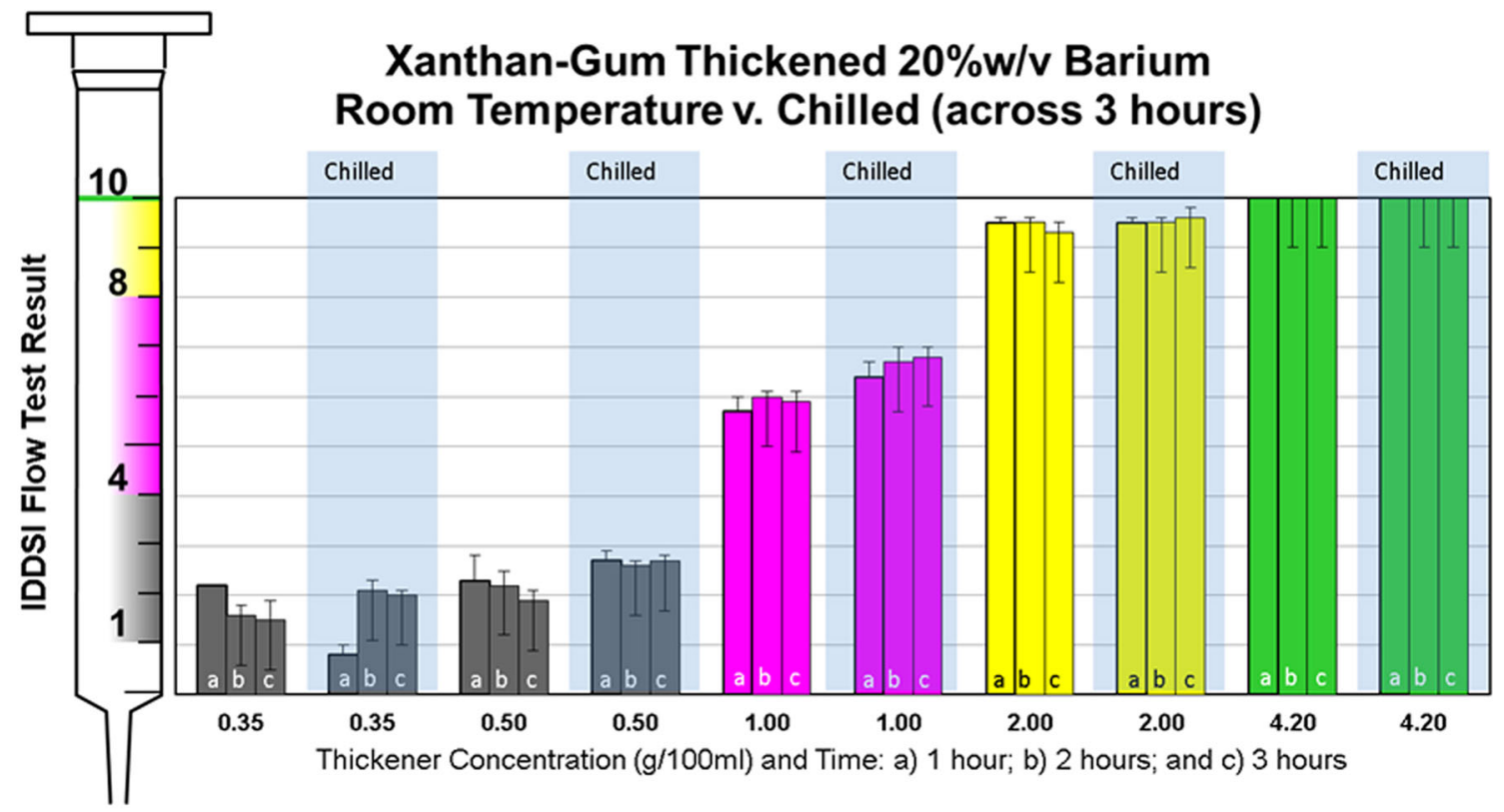

Fig. 3 IDDSI Flow Test results for room-temperature and chilled xanthan-gum-thickened barium over $3 \mathrm{~h}$

Table 1 Final recipes $(\mathrm{g} / 100 \mathrm{ml})$ for all non-barium and barium liquids by IDDSI level

\begin{tabular}{llllll}
\hline IDDSI level & \multicolumn{2}{l}{ Non-barium } & & & Barium \\
\cline { 2 - 3 } & Xanthan gum & Starch & & Xanthan gum & Starch \\
\hline 1 & 0.65 & 4.15 & & 0.4 & 2.85 \\
3 & 1.25 & 4.77 & & 1.02 & 3.75 \\
3 & 2.1 & 5.85 & & 2.2 & 5.1 \\
\hline
\end{tabular}

continuum is much lower when using xanthan-gum- rather than starch-based thickeners. For example, Fig. 1a and $b$ show non-barium liquids thickened with the starch and xanthan-gum thickeners, respectively. In both graphs, the first data point shows a slightly thick liquid with an average of $1.4 \mathrm{ml}$ left in the syringe after $10 \mathrm{~s}$ of flow. For the starch-thickened liquid (Fig. 1a), $4.1 \mathrm{~g} / 100 \mathrm{ml}$ of thickener was required to reach this level; the corresponding xanthan-gum-thickened liquid (Fig. 1b) required only $0.65 \mathrm{~g} /$ $100 \mathrm{ml}$ of thickener to achieve the same flow. The common 
trend for thickener labels to specify the amount of thickener needed using a simplified 1-, 2-, or 3-scoop methodology obscures this difference. Users may not be aware that the size of the scoop included in a package of thickener has been adjusted to compensate for the different concentrations required across thickener types.

Several of the recipes that were tested in this study yielded IDDSI Flow Test results of $10 \mathrm{ml}$ (i.e., no drip). It is important to mention that this result represents saturation of the IDDSI Flow Test. Confirmation that a liquid falls in the IDDSI extremely thick liquid category requires supplementary spoon tilt and fork drip tests as per IDDSI guidelines. It is acknowledged that these supplementary tests are subjective and do not permit precise matching of flow characteristics across liquids within this level.

In some cases, the recipes in this study produced flow test results that fell close to the boundaries of the IDDSI levels. For example, in Fig. 1b, $1.0 \mathrm{~g} / 100 \mathrm{ml}$ of xanthangum thickener added to water produced a liquid with an average flow test result of $4.5 \mathrm{ml}$, just above the lower boundary of the IDDSI mildly thick range. In such cases, our data show that small changes in thickener concentration can shift the liquid closer to the center of the target IDDSI range. In the example cited, the addition of $0.3 \mathrm{~g}$ of xanthan gum $(1.3 \mathrm{~g} / 100 \mathrm{ml})$ moved the flow test result to the middle of the mildly thick range at $6.1 \mathrm{ml}$. Until clearer evidence regarding the clinical significance of small differences in liquid flow is available [2] we recommend that clinicians target flow test results closer to the middle of each IDDSI range.

The reconstitution of barium sulfate powders with water and thickening agents is a widespread but off-label practice that has arisen due to restricted access to pre-thickened barium products outside the United States. The variations in flow that were seen across small differences in thickener concentration in this study underscore the need to follow recipes when preparing barium for videofluoroscopy. Clinicians should not assume that addition of the same amount of thickener to a non-barium liquid and a barium suspension will result in liquids with similar flow. For example, in Fig. $1 \mathrm{~b} 1.0 \mathrm{~g} / 100 \mathrm{ml}$ of xanthan-gum thickener added to water resulted in an average flow test result of $4.4 \mathrm{ml}$ at the 1-h timepoint post mixing; by contrast, Fig. 1d shows that the same amount of xanthan-gum thickener $(1.0 \mathrm{~g} / 100 \mathrm{ml})$ in a $20 \% \mathrm{w} / \mathrm{v}$ barium suspension resulted in a thicker liquid with a flow test result of $5.7 \mathrm{ml}$. Despite this difference, both of these results fall in the IDDSI mildly thick range; it is not yet known whether a flow difference of this magnitude has clinical significance. However, more dramatic differences are seen with the starch-based thickener. As seen in Fig. 1a, $5 \mathrm{~g} / 100 \mathrm{ml}$ of starch-based thickener in water yields a mildly thick liquid with a flow test result of $4.9 \mathrm{ml}$. However, a similar amount of the same thickener (Fig. 1c; $5.1 \mathrm{~g} / 100 \mathrm{ml}$ ) added to a $20 \% \mathrm{w} / \mathrm{v}$ barium suspension resulted in a moderately thick liquid with a flow test result of $9.3 \mathrm{ml}$. These examples are consistent with previous literature [21-23] showing that the combination of barium and thickening agents may alter multiple rheological parameters, and specifically may lead to further thickening. In addition, it must be emphasized that the barium stimuli in this study were prepared in a low concentration $(20 \% \mathrm{w} / \mathrm{v})$; one might expect that the addition of thickener to higher concentrations of barium may lead to even greater thickening. The reader should be cautioned that the interactions illustrated in this study are likely to be specific to the particular brands of barium suspension and/or thickener studied, based on their composition, and the patterns seen cannot be generalized to other products.

When comparing room-temperature liquids to liquids that were chilled, we found very few differences in flow. All of the barium-based recipes yielded stable IDDSI Flow Test results over time, up to $3 \mathrm{~h}$. The addition of refrigeration as a variable had very little impact. As seen in Fig. 2, only one barium-thickened liquid recipe ( $3.3 \mathrm{~g} /$ $100 \mathrm{ml}$ starch) showed temperature-related thickening which crossed the boundary from slightly to mildly thick for the chilled product.

\section{Limitations}

This study is not without limitations. The thickeners tested are limited to two particular products, mixed with a single commercially available barium sulfate suspension. The results may not be generalizable to other products. Furthermore, these products were reconstituted with commercially available bottled waters. In clinical practice, offlabel use of barium may involve reconstitution with other liquids, such as infant formula or breastmilk [24, 25]. Clinicians should be cautioned that the kinds of interactions seen between thickeners and barium in the current study may also occur when thickeners or barium is added to other liquids, based on the protein or macronutrient composition of the liquid [16]. In such cases, the IDDSI Flow Test provides a means of checking flow and confirming similarity between the barium and non-barium product.

\section{Conclusions}

A practical goal of this study was to determine recipes for matched non-barium and barium stimuli, which would fall clearly within the slightly to moderately thick levels of the IDDSI framework and remain in those levels over a 3-h 
timeframe post mixing at room temperature. This goal was achieved. As a rule, the combination of barium powder and thickener led to additional thickening compared to thickened liquids prepared without barium. Adjustments to the thickener amounts were required to correct for this interaction, particularly when using starch-based thickeners.

Funding Funding for this study was provided through an RO1 grant from the National Institute on Deafness and Other Communication Disorders DC011020 to the second author.

\section{Compliance with Ethical Standards}

Conflicts of interest The first author received scholarship funding from the University of Toronto, Toronto Rehabilitation Institute Student Scholarship (Ontario Student Opportunity Trust Funds; OSOTF). The second author (CMS) serves on the board of directors for the International Dysphagia Diet Standardisation Initiative, which established the gravity-flow levels for liquid classification that were used in this study. The second author has also served as an expert panelist and principal investigator for a different study conducted by Nestlé Health Science, the manufacturer of the thickeners used in this study. The second author has also previously received an educational grant from Bracco Canada, the manufacturer of the barium products used in this study. Nestlé Health Science and Bracco Canada were not involved in the research described in this manuscript.

Ethics Approval This study complies with ethical standards. An ethics submission was not required as this project did not involve human or animal subjects.

Open Access This article is distributed under the terms of the Creative Commons Attribution 4.0 International License (http://creative commons.org/licenses/by/4.0/), which permits unrestricted use, distribution, and reproduction in any medium, provided you give appropriate credit to the original author(s) and the source, provide a link to the Creative Commons license, and indicate if changes were made.

\section{References}

1. Garcia JM, Chambers EIV, Molander M. Thickened liquids: practice patterns of speech-language pathologists. Am J SpeechLang Pathol. 2005;14:4-13.

2. Steele CM, Alsanei WA, Ayanikalath S, et al. The influence of food texture and liquid consistency modification on swallowing physiology and function: a systematic review. Dysphagia. 2015;30:2-26.

3. Cichero JAY, Steele C, Duivestein J, et al. The need for international terminology and definitions for texture-modified foods and thickened liquids used in dysphagia management: foundations of a global initiative. Curr Physical Med Rehabil Rep. 2013;1:280-91.

4. Fink TA, Ross JB. Are we testing a true thin liquid? Dysphagia. 2009;24:285-9.

5. Nita SP, Murith M, Chisholm H, et al. Matching the rheological properties of videofluoroscopic contrast agents and thickened liquid prescriptions. Dysphagia. 2013;28:245-52.
6. Glassburn DL, Deem JF. Thickener viscosity in dysphagia management: variability among speech-language pathologists. Dysphagia. 1998;13:218-22.

7. Cichero JAY, Jackson O, Halley PJ, et al. How thick is thick? Multicenter study of the rheological and material property characteristics of mealtime fluids and videofluoroscopy fluids. Dysphagia. 2000;15:188-200.

8. Curran J, Groher ME. Development and dissemination of an aspiration risk reduction diet. Dysphagia. 1990;5:6-12.

9. Coster ST, Schwarz WH. Rheology and the swallow-safe bolus. Dysphagia. 1987;1:113-8.

10. Hanson B, O'Leary MT, Smith $\mathrm{CH}$. The effect of saliva on the viscosity of thickened drinks. Dysphagia. 2012;27:10-9.

11. Dewar RJ, Joyce MJ. Time-dependent rheology of starch thickeners and the clinical implications for dysphagia therapy. Dysphagia. 2006;21:264-9.

12. Steele CM, Van Lieshout PHHM, Goff D. The rheology of liquids: a comparison of clinicians? Subjective impressions and objective measurement. Dysphagia. 2003;18:182-95.

13. Garcia JM, Et Chambers, Matta Z, et al. Serving temperature viscosity measurements of nectar- and honey thick liquids. Dysphagia. 2008;23:65-75.

14. Garcia JM, Et Chambers, Matta Z, et al. Viscosity measurements of nectar- and honey thick liquids: product, liquid, and time comparisons. Dysphagia. 2005;20:325-35.

15. Sopade PA, Halley PJ, Cichero JAY, et al. Physical properties of thickened fluids used in dysphagia management. Asia Pac J Clin Nutr. 2006;15:S137.

16. Hadde EK, Nicholson TM, Cichero JAY, et al. Rheological characterisation of thickened milk components (protein, lactose and minerals). J Food Eng. 2015;166:263-7.

17. American Dietetic Association National Dysphagia Diet Task Force. National dysphagia diet: standardization for optimal care. Chicago; 2002.

18. Cichero JAY, Lam P, Steele CM, et al. Development of international terminology and definitions for texture-modified foods and thickened fluids used in dysphagia management: the IDDSI framework. Dysphagia. 2017;32:293-314.

19. Hanson B. A review of diet standardization and bolus rheology in the management of dysphagia. Curr Opin Otolaryngol Head Neck Surg. 2016;24:183-90.

20. Pelletier CA, Dhanaraj GE. The effect of taste and palatability on lingual swallowing pressure. Dysphagia. 2006;21:121.

21. Nita SP, Murith M, Chisholm H, et al. Matching the rheological properties of videofluoroscopic contrast agents and thickened liquid prescriptions. Dysphagia. 2013;28(2):245-52.

22. Steele CM, Molfenter SM, Peladeau-Pigeon M, et al. Challenges in preparing contrast media for videofluoroscopy. Dysphagia. 2013;28:464-7.

23. Ekberg O, Bulow M, Ekman S, et al. Effect of barium sulfate contrast medium on rheology and sensory texture attributes in a model food. Acta Radiol. 2009;50:131-8.

24. Cichero J, Nicholson T, Dodrill P. Liquid barium is not representative of infant formula: characterisation of rheological and material properties. Dysphagia. 2011;26:264-71.

25. Frazier J, Chestnut AH, Jackson A, et al. Understanding the viscosity of liquids used in infant dysphagia management. Dysphagia. 2016;31:672-9.

\section{Carly E. A. Barbon MA}

Catriona M. Steele PhD 